\title{
Transition Probabilities of Disablement for Malaysian Population in Need of Long-Term Care
}

\author{
S.N. Shair ${ }^{1 *}$ and T.S. Purcal ${ }^{2}$ \\ ${ }^{l}$ Centre for Actuarial Studies, Faculty of Computer and Mathematical Sciences, Universiti \\ Teknologi MARA, Shah Alam, Selangor Malaysia \\ ${ }^{2}$ Centre for Risk Analytics, Department of Actuarial Studies and Business Analytics, \\ Macquarie Business School, Macquarie University, Sydney, Australia
}

\begin{abstract}
This research aims to estimate the transition probabilities of lives becoming disabled and the extent to which they are disabled using the approach of functional Markov model. The transition probability from one disabled state to a more or less severely disabled state is best estimated using longitudinal data in which the change in the health status of each respondent can be monitored over one or more years. Such data are limited in Malaysia, typically covering only a smaller area of the nation. The functional Markov model overcomes such data limitations, using cross-sectional data which measures the disability status of individuals only at one point in time and build a functional form for the transition probabilities in a multiple state model. Results suggested that multiple state model's prevalence rates replicated the Malaysian prevalence rates quite well, indicating that the parameters of the probability of deterioration had been estimated accurately with sum squared of errors less than $5 \%$ for almost all age groups and disability levels. Furthermore, severely disabled elderlies, especially among the oldest age group, have the highest probability to die compared to less severely disabled elderlies.
\end{abstract}

Keywords: long term care; disability; transition rates; multiple state model

\section{INTRODUCTION}

Population ageing affects long term care systems, particularly in terms of demand and its associated costs. Malaysian mortality and fertility are projected to decrease over the next few decades, exacerbating population ageing. Goh \& Lai (2013) argues Malaysia will become an aged society by 2030, that is when older adults aged 60 years and above reach $15 \%$ of the total population. These changes are likely to have a profound impact on the demand for long term care due to the higher prevalence of disability amongst the elderly (Goh \& Lai 2013, Pollard 1996).

Since the provision of long-term care is costly, an accurate estimation of transition probabilities of disablement and its extent is important, to avoid underestimation or overestimation of long-term care costs. Generally, the estimation of transition probabilities across disability states (for example, a healthy person becoming disabled or a moderately disabled person becoming severely disabled, and so on) requires national longitudinal data, which records the disability status of an individual at two points in time using two consecutive surveys (Hariyanto et al., 2013). Nevertheless, longitudinal data is often unavailable, or limited to a smaller size population, in many countries, including Malaysia. One may use other countries' comprehensive longitudinal survey data, such as the National Long-Term Care Survey in the U.S. (Manton, 2010). Still, it may well misrepresent the disability status of the Malaysian population.

To address limitations of longitudinal survey data, Rickayzen \& Walsh (2002) developed a method for estimating the transition probabilities of disablement using cross-sectional data which measures the disability status of individuals only at one point in time. They built a functional form for the transition probabilities in a multiple state model. Parameters in the model are estimated in such a way

*Corresponding author’s e-mail: syazreen@uitm.edu.my 
that the initial prevalence rates are replicated over one year. Leung (2004) adopted the Rickayzen \& Walsh (2002) model to project the likely number of people requiring long term care using Australia data and proposed some modifications. Instead of using age 20 as the beginning age, his model covers all ages to allow disabilities that may have arisen at earlier ages. Leung (2004) also proposed different functions for mortality and disability improvement using a method from the Continuous Mortality Investigation Bureau (1999). Also, the application was extended to estimate associated long-term care costs.

Hariyanto et al. (2013) also adopted the same model framework to estimate the transition probabilities across levels of disability for Australia. As opposed to Leung (2004), who used 1998 data, Hariyanto et al. (2013) used more recent 2003 data. Interestingly, the model made use of two consecutive available disability prevalence rates in 1998 and 2003 to obtain the prevalence rates in every year from 1999 to 2002. Bueno (2013) showed that the same model applies to a developing country, Brazil. However, several formula and parameters were adjusted to accommodate Brazilian disability data. Research from Omar, Shair \& Asmuni (2020) proposed the cancer stage transition model for lung cancer patients in Malaysia using the same approach. However, the estimated rates were in terms of overall and did not differ by age groups and gender.

This research is the first to estimate the transitions probabilities of disablement for the Malaysian population according to different disability states, age and gender. We adopt the functional Markov Chain framework from Rickayzen \& Walsh (2002) and Leung (2004). This paper extends the models, for the case of Malaysian disability in which the parameter values will be estimated and reflect Malaysian disability experience. We propose four disability levels, including able, mild, moderate and severe, to estimate the transition disablement.

This paper is organised as follows. Section II describes the age and gender-specific disability prevalence data, while Section III explains the extension of the functional Markov model to suit Malaysian disability data. Section IV provides results and discussions of study. Finally, Section V concludes and provides possible future directions.

\section{DATA}

Malaysian disability data can be obtained from the National Health Morbidity Survey (NHMS), which is conducted once in every four years. Unfortunately, at the time we conducted this research, data according to disability levels by age groups were not available from the survey report. These age-specific data were necessary to estimate the transition probabilities across disability levels by ages.

To overcome the limitations of the NHMS data, we used the WHS (2002), conducted by the World Health Organization (WHO). This WHS survey included many countries, including Malaysia. For Malaysia, the survey was carried out in all states of Malaysia, which consists of 6,037 samples of Malaysians over the age of 18 . The data is valid, reliable and comparable source of international health data, describing characteristics of individual health and health systems.

There are nine main sets of questions; however, we gather data from which refers to the individual's health state description. Five possible answers for every question were set as 1 is very good, 2 is good, 3 is moderate, 4 is bad, and 5 is very bad. We recode the answer to represent disability levels such that 1 is "able", 2 is "mild", 3 is "moderate" and as data for 5 are very small, we combine these with 4 and recode as "severe".

To estimate the prevalence rates of disability for Malaysia, we collected data primarily from the following three questions of WHS (2002):

i. q2010: "Overall in the last 30 days, how much diffIculty did you have with moving around?",

ii. q2020: "Overall in the last 30 days, how much diffIculty did you have with self care, such as washing or dressing yourself?",

iii. q2050: "Overall in the last 30 days, how much diffIculty did you have with concentrating or remembering things?".

The above three questions were chosen so that our disability data describes the prevalence of people in need of long-term care services. Following Chan et al. (2004), we describe people requiring long term care are those with mobility and self-care limitations or with cognitive impairment. Data for each question were aggregated according to age group, gender and disability level and then divided by the total exposure to estimate the prevalence rates per 1,00o. The prevalence rates of the three questions were then averaged to obtain the prevalence rates for Malaysia by age and severity level, as 
shown in Table 1 and Table 2 for males and females, respectively.

Table 1. Malaysian age-specific disability prevalence rates per 1000 males estimated from WHS (2002)

\begin{tabular}{|l|l|l|l|l|}
\hline Age & Able & Mild & Moderate & Severe \\
\hline 18 & 904.42 & 81.15 & 11.52 & 2.91 \\
\hline 25 & 878.04 & 100.75 & 18.24 & 2.98 \\
\hline 30 & 868.32 & 101.59 & 22.95 & 7.14 \\
\hline 35 & 875.40 & 104.21 & 18.78 & 1.61 \\
\hline 40 & 882.74 & 97.52 & 13.46 & 6.27 \\
\hline 45 & 873.57 & 88.92 & 27.88 & 9.63 \\
\hline 50 & 834.10 & 130.95 & 29.12 & 5.83 \\
\hline 55 & 801.19 & 140.03 & 42.38 & 16.73 \\
\hline 60 & 772.90 & 153.17 & 53.66 & 20.27 \\
\hline 65 & 659.21 & 233.69 & 76.51 & 30.58 \\
\hline 70 & 594.94 & 258.81 & 114.01 & 32.24 \\
\hline 75 & 569.10 & 275.37 & 90.16 & 65.36 \\
\hline 80 & 476.61 & 290.14 & 122.96 & 110.29 \\
\hline
\end{tabular}

Table 2. Malaysian age-specific disability prevalence rates per 1000 females estimated from WHS (2002)

\begin{tabular}{|l|l|l|l|l|}
\hline Age & Able & Mild & Moderate & Severe \\
\hline 18 & 906.70 & 83.01 & 7.95 & 2.74 \\
\hline 25 & 899.42 & 84.58 & 13.48 & 2.52 \\
\hline 30 & 866.23 & 103.57 & 26.32 & 3.88 \\
\hline 35 & 870.77 & 101.09 & 26.09 & 2.05 \\
\hline 40 & 876.73 & 105.11 & 11.59 & 6.57 \\
\hline 45 & 881.22 & 74.44 & 32.36 & 11.97 \\
\hline 50 & 809.04 & 143.37 & 43.89 & 3.70 \\
\hline 55 & 779.81 & 146.48 & 51.87 & 22.85 \\
\hline 60 & 716.96 & 196.36 & 67.15 & 19.53 \\
\hline 65 & 583.61 & 279.21 & 89.73 & 47.45 \\
\hline 70 & 553.53 & 264.12 & 147.65 & 34.71 \\
\hline 75 & 466.40 & 345.16 & 133.00 & 55.44 \\
\hline 80 & 518.25 & 238.10 & 101.65 & 142.00 \\
\hline
\end{tabular}

The estimated WHS (2002) prevalence rates from Table 1 and Table 2 show that the disability rates are varied by age, disability level and gender. The healthy (able) rates decrease as age increases, and the disability (mild, moderates and severe) prevalence rates generally increase as age increases. These trends appear in both males and females. The increase in disability prevalence rates by age for both males and females are considerable for the severe group such that it increases more than triples between age 55 and 75 years old.
A comparison between gender shows that females' prevalence rates for mild, moderate and severe categories are higher than that of males for age 50 years and above, but lower for ages below 50. This indicates that Malaysian elderly females suffered from disabilities, in higher percentages, compared to elderly males. The result is consistent with disability patterns of elderly people in Bangladesh (Tareque et al., 2017).

\section{METHODS}

The estimation of transition probabilities between several disability states followed the multiple state modelling framework from Rickayzen \& Walsh (2002). Some formulae and parameters were adjusted accordingly to Malaysian data. The model was applied to the Malaysian prevalence rate of disability according to age, gender and disability levels.

We proposed four disability states, namely able, mild, moderate and severe, and one absorbing state, death, in the model. Each disability state was denoted by $j: j=$ $0,1,2,3$ where 0 represented the able or healthy state, 1 was mild, 2 was moderate, and 3 was severe.

There were three types of transition probabilities included in the model, including death probabilities, deterioration probabilities (probabilities of moving to any worse disability state) and improvement probabilities (probabilities of recovering to a less disabled state or able state). Similar to Rickayzen \& Walsh (2002), Leung (2004) and Hariyanto et al. (2013), the following assumptions were adopted in the model: i. deterioration to any worse disability state was allowed over a year; ii. improvement was only allowed by one category; iii. only one transition was possible over one year. See Figure 1 for the proposed model of disability transition states for the Malaysian population.

The parameters of the equation for deterioration probabilities were estimated through the fitting process. In contrast, death and improvement probabilities were determined separately and included as an input in the fitting process. The deterioration probabilities were found by assuming a functional form for the probabilities in the multiple state model. The parameters were estimated such that the model's prevalence rates replicate the WHS estimated prevalence rates reported in Table 1 and Table 2. 
We assume stationary population characteristics when deriving transition probabilities.



Figure 1. The Proposed Four-State with Absorbing State Markov Chain model of disability for the Malaysian population

Similar to Leung (2004), the observed prevalence rates were replicated using the optimisation procedure available in Excel's 'Solver' function so that the sum of the squared difference of the calculated WHS prevalence rates and the model's prevalence rates were minimised. The mathematical formulae used to estimate the probability of death, probability of deterioration and probability of improvement are discussed in the following sub-sections.

\section{A. The Probability of Death}

The probability of death can be divided into two categories, overall mortality and additional mortality. The overall mortality assumes that people who are in the same age group and gender will experience the same mortality experience, regardless of their level of disability. In contrast, the additional mortality imposes an extra rate on those who are in the severe disability category $(j=3)$. This translates to the mortality rates of disabled people get higher as disability status becomes more severe. The probability of death is given as follows;

$$
\operatorname{Mort}(x, j)=\operatorname{Overall}(x)+\operatorname{Additional}(x, j)
$$

where $\operatorname{Mort}(x, j)$ is the one-year death probability that applies to an individual aged $x$ in disability state $j(j=$ $0,1,2,3)$. The $\operatorname{Overall}(x)$ is the one-year death probability which applies to any individual aged $x$ regardless of his/her disability status. These probabilities were obtained from the Department of Statistics Malaysia (2002). The $\operatorname{Additional}(x, j)$ is the additional death probability imposed on people who are in the severe disability state which is $j=3$ and is given by;

$$
\operatorname{Additional}(x, j)=\frac{M}{1+1.1^{50-(x+0.5)}} \times \frac{\max (j-2,0)}{2}
$$

where $M$ is the maximum additional annual mortality imposed on severely disabled persons, there is a little known about the impact of disability on mortality in Malaysia throwing up some uncertainties. Rickayzen \& Walsh (2002) used $M=0.2$ for the U.K. population whereas Leung (2004) used $M=0.15$ for Australia, that followed the value suggested by Society of Actuaries Long-Term Care Valuation Insurance Methods Task Force (1995). Due to the limited information regarding the relationship between the disability and death in Malaysia we used M values from Hariyanto et al. (2013) for Australia which are M of 0.11 for males and 0.08 for females.

\section{B. The Probability of Deterioration}

The estimation of deterioration probabilities includes two types of deterioration. First, a healthy person becomes disabled, denoted as Deteriorate $(x, 0, j)$ and second, a disabled person deteriorates to a more severely disabled state, denoted as Deteriorate $(x, m, n)$. The formula for the first, which is the probability a healthy person $(j=0)$ aged $x$ deteriorates to any disability category $\mathrm{j}(\mathrm{j}=1,2,3)$, comprises two components, expressed as below;

$$
\operatorname{Deteriorate}(x, 0, j)=\operatorname{Newdis}(x) \times \operatorname{Severity}(x, j)
$$

The first component, Newdis $(x)$, represents the probability of a healthy person aged $x$ becoming disabled for the first time, which can be estimated as follows. 
$\operatorname{Newdis}(x)=\alpha\left[\left(A+\frac{D-A}{1+B^{C-x}}\right) \times\left(1-\frac{1}{3}\left(-\left(\frac{x-E}{4}\right)^{2}\right)\right)\right]$

(4)

For Malaysian data, we apply the same formula for both males and females. The $A$ parameter is the limit of probability of becoming disabled at young ages and $D$ is the limit of probability of becoming disabled that would apply at extremely high ages. Parameters $B$ and $C$ determine how rapidly the probabilities change between the two extreme values, $A$ and $D$. Age $E$ identifies the kink in the new disabled rates after which disability rates increase dramatically.

The second component, Severity $(x, j)$, is the probability that a healthy person aged $x$ deteriorates to a particular disabled state given that he becomes disabled over the year. The formula for $\operatorname{Severity}(x, j)$ is;

$$
\operatorname{Severity}(x, j)=\frac{W(j) \times f(x)^{j-1}}{\operatorname{Scale}(x)}
$$

where

$$
f(x)=P+\frac{1-P}{1+Q^{R-x}}
$$

and

$$
\operatorname{Scale}(x)=\sum_{j=1}^{3} W(j) f(x)^{j-1}
$$

$W(j)$ are category widths designed to allow for some categories having more people than others. Variables $P, Q$ and $R$ reflect the age-dependence of disability and $\operatorname{Scale}(x)$ ensures the probabilities sum to one.

The second type of deterioration probability, after that discussed in equation (3), is Deteriorate $(x, m, j)$, which is the probability that a disabled person in disability state $m$ becomes more severely disabled--- moves to state $j, j>m$ as follows;

$$
\operatorname{Deteriorate}(x, m, j)=\operatorname{Deteriorate}(x, 0, j) \times I^{m}
$$

Equation (8) shows that the probability of a person at disability level $m$ deteriorating to level $\mathrm{j} j(j>m)$ is $I^{m}$ times the probability of that person from the healthy state deteriorates to disability level $j$.

\section{The Probability of Improvement}

As in Rickayzen \& Walsh (2002), Leung (2004) and Hariyanto et al. (2013), our model includes the probability that a person who is disabled can improve his disability condition to a less severe state or the able state. A simple assumption has been adopted in which the improvement in the disability is allowed to only one less severe category over a year, if and only if he survives and does not deteriorate to a more severe disability state. Rickayzen \& Walsh (2002) used a constant of $10 \%$ as the improvement rate for every disability category over a year. This paper, however, follows Leung (2004) in allowing a variable chance of improvement from different disability states as in Table 3 .

Table 3. The estimated parameters for Newdis $(x)$ the formula for males and females

\begin{tabular}{|l|c|c|c|}
\hline Improvement & $\begin{array}{c}\text { Mild to } \\
\text { able }\end{array}$ & $\begin{array}{c}\text { Moderate to } \\
\text { mild }\end{array}$ & $\begin{array}{c}\text { Severe to } \\
\text { moderate }\end{array}$ \\
\hline Recovery rate & $15.0 \%$ & $15.0 \%$ & $12.5 \%$ \\
\hline
\end{tabular}

\section{Projection Method}

To begin the one-year projections, the initial number of populations in each category is estimated such that the male and female disability prevalence rates of the model year 2002 are multiplied by the population number from DoSM by agegroup, males and females of the same year. Although the model prevalence rates are not fully consistent with the data prevalence rates, the differences are assumed to be minimal. Below we outline the method used to project the age-sexspecific population by disability levels. Define $\operatorname{Lives}(x, t, n)$ as the number of lives aged $x$ in year $t$ in disability category $n$. Then;

$$
\begin{aligned}
& \text { Lives }(x, t, n)=\text { Lives }(x-1, t-1, n) \\
& \qquad \times[1-\operatorname{Mortality}(x-1, t-1, n)] \\
& \times[1-\text { Deteriorate_From }(x-1, t-1, n)] \\
& \times\left[1-\operatorname{Improve\_ From~}(x-1, t-1, n)\right] \\
& + \text { Deteriorate_To }(x, t, n) \\
& + \text { Improve_To }(x, t, n)
\end{aligned}
$$

where $\operatorname{Mortality}(x, t, n)$ is the probability that a person aged $x$ at time $t$ and with disability category $\mathrm{n}$ dies in the following 
year which defined as $\operatorname{Mortality}(x, t, n)=\operatorname{Overall}(x, t)+$ $\operatorname{Additional}(x, t, n)$. Whereas Deteriorate_From $(x, t, n)$ is the probability that a person aged $x$ at time $t$ and with disability category $\mathrm{m}$ makes a transition to a more severe disability category which defined as;

$$
\operatorname{Deteriorate\_ } \operatorname{From}(x, t, n)=\sum_{n=m+1}^{3} \operatorname{Deteriorate}(x, t, m, n)
$$

Also, Improve_From $(x, t, n)$ is the probability that a person, who does not deteriorate to a more severe disability category, improves by one category. Thus;

$$
\text { Improve_From }(x, t, n)=\left\{\begin{array}{rl}
0.15, & n=1,2 \\
0.125 & n=3
\end{array}\right.
$$

Further, Deteriorate_To $(x, t, n)$ is the number of persons aged $x$ at time $t$ who make a transition to disability category $n$ from a less severe disability category. Thus;

Deteriorate_To $(x, t, n)$

$$
\begin{aligned}
& =\sum_{m=0}^{n-1} \operatorname{Lives}(x-1, t-1, n) \\
& \times[1-\operatorname{Mortality}(x-1, t-1, m)] \\
& \times \operatorname{Deteriorate}(x-1, t-1, m, n)
\end{aligned}
$$

Finally, Improve_To $(x, t, n)$ is the number of persons aged $x$ at time $t$ who makes a transition to disability category $\mathrm{n}$ from a more severe disability category $n+1$. Thus,

Improve_To $(x, t, n)$

$$
\begin{aligned}
& =\operatorname{Lives}(x-1, t-1, n+1) \\
& \times[1-\operatorname{Mortality}(x-1, t-1, n+1)] \\
& \times[1 \\
& - \text { Deteriorate_From }(x-1, t-1, n+1)] \\
& \times\left[1-\operatorname{Improve\_ From}(x-1, t-1, n+1)\right]
\end{aligned}
$$

\section{RESULTS AND DISCUSSION}

As mentioned previously, transition probabilities are estimated by assuming a functional form for the transition probabilities in the Markov model and subsequently finding parameters for each function such that the initial prevalence rates are replicated over 1 year. From the optimisation procedure, the sum of the squared difference of the prevalence rates in the data and the corresponding prevalence rates from the model were minimised.

Table 4. The sum of the squared difference (data minus model, as a percentage (\%)) for males

\begin{tabular}{|c|c|c|c|c|}
\hline Age & Able & Mild & Moderate & Severe \\
\hline 18 & 3.42 & -1.91 & -0.99 & -0.52 \\
\hline 25 & -0.78 & 1.27 & -0.12 & -0.38 \\
\hline 30 & -1.65 & 1.25 & 0.34 & 0.05 \\
\hline 35 & -0.06 & 0.83 & -0.22 & -0.55 \\
\hline 40 & 2.19 & -0.97 & -1.04 & -0.18 \\
\hline 45 & 3.29 & -3.29 & -0.01 & 0.01 \\
\hline 50 & 0.70 & 0.00 & -0.22 & -0.48 \\
\hline 55 & -0.08 & -0.77 & 0.52 & 0.37 \\
\hline 60 & 2.77 & -3.09 & 0.25 & 0.07 \\
\hline 65 & -2.23 & 1.44 & 0.66 & 0.12 \\
\hline 70 & -1.67 & 0.83 & 2.04 & -1.20 \\
\hline 75 & 3.56 & -0.04 & -3.29 & -0.22 \\
\hline 80 & 0.19 & 0.01 & -2.32 & 2.05 \\
\hline & & & &
\end{tabular}

Table 5 . The sum of the squared difference (data minus model, as a percentage (\%)) for females

\begin{tabular}{|c|c|c|c|c|}
\hline Age & Able & Mild & Moderate & Severe \\
\hline 18 & 4.89 & -2.14 & -2.11 & -0.60 \\
\hline 25 & 1.87 & -0.39 & -1.03 & -0.45 \\
\hline 30 & -1.31 & 1.35 & 0.26 & -0.30 \\
\hline 35 & 0.33 & 0.22 & 0.00 & -0.55 \\
\hline 40 & 1.81 & -0.01 & -1.65 & -0.15 \\
\hline 45 & 3.39 & -3.89 & 0.17 & 0.33 \\
\hline 50 & 0.56 & -0.01 & 0.29 & -0.84 \\
\hline 55 & 2.60 & -2.89 & -0.29 & 0.59 \\
\hline 60 & 1.63 & -0.90 & -0.37 & -0.36 \\
\hline 65 & -6.10 & 4.53 & -0.01 & 1.59 \\
\hline 70 & -2.90 & 0.58 & 3.40 & -1.08 \\
\hline 75 & -4.32 & 8.29 & -1.18 & -2.79 \\
\hline 80 & 6.87 & -0.44 & -6.43 & 0.00 \\
\hline
\end{tabular}

We can see from the Table 4 and Table 5 that the percentage errors for males and females appear relatively small, less than $5 \%$, across almost all ages and disability states, except for the oldest age group among females. Higher errors can only be seen for females aged 70 years old and above. This result is consistent with Leung (2004) who found poor fit for age 85 and above for Australian data. 


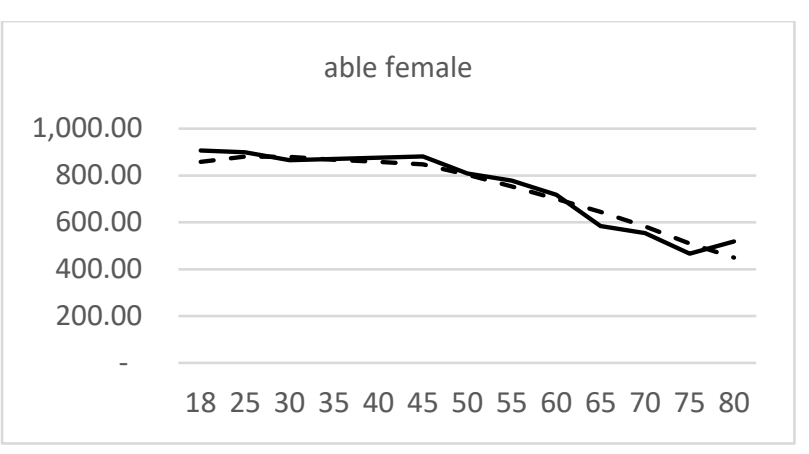

(a)

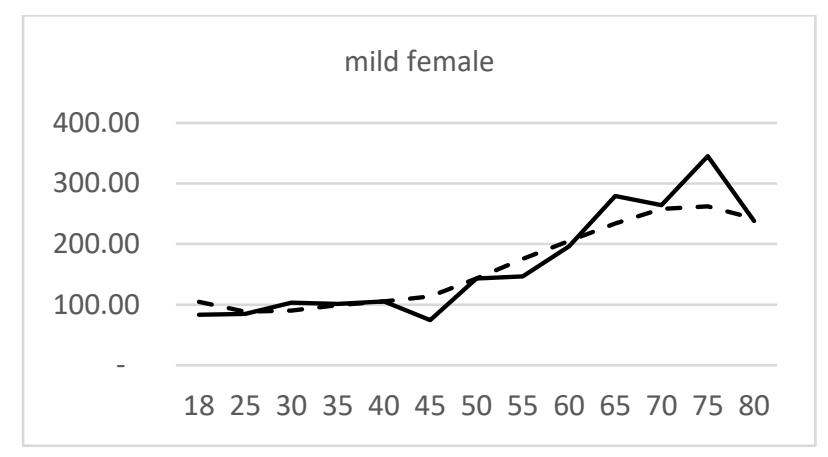

(b)

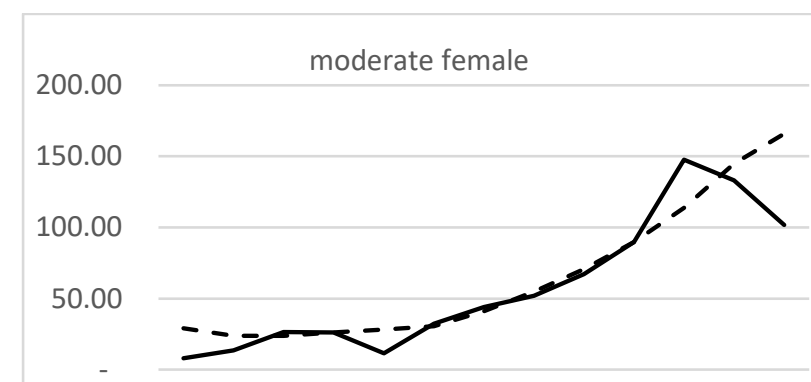

$\begin{array}{lllllllllllll}18 & 25 & 30 & 35 & 40 & 45 & 50 & 55 & 60 & 65 & 70 & 75 & 80\end{array}$

(c)

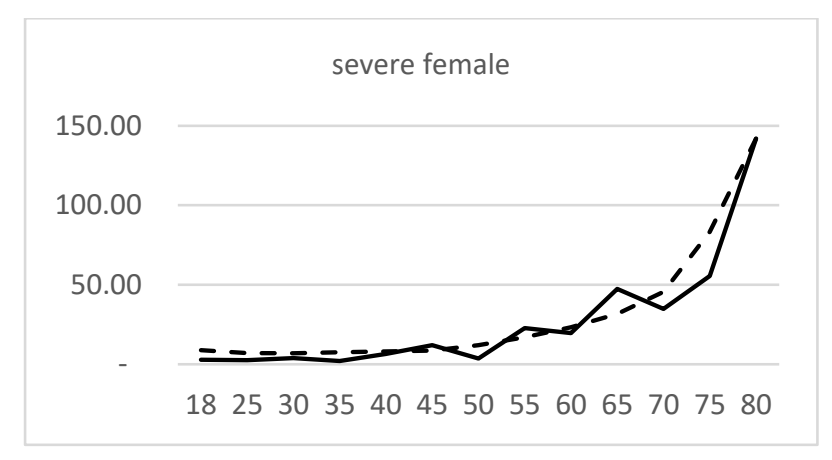

(d)

Figure 2. The comparison between the calculated WHS disability prevalence rates (solid lines) and the model's prevalence rate (dashed lines) for females with different disability levels: able (a), mild (b), moderate (c) and severe (d)

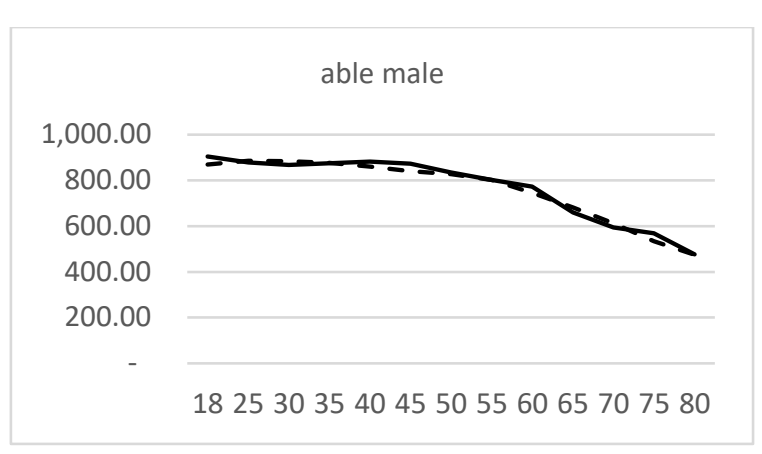

(a)

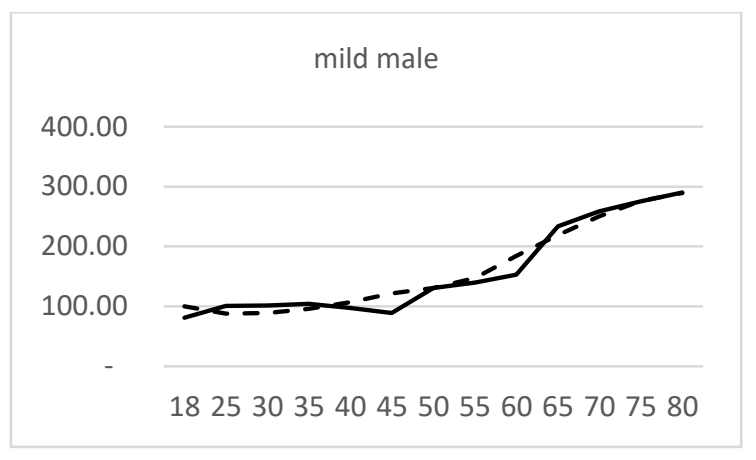

(b)

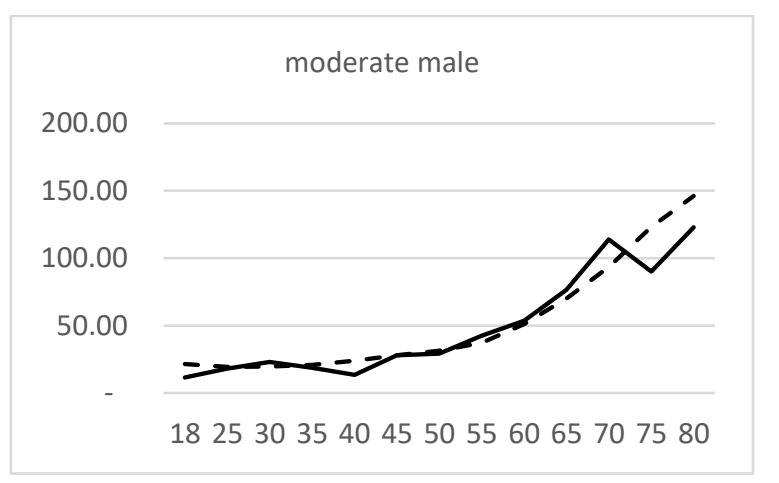

(c)

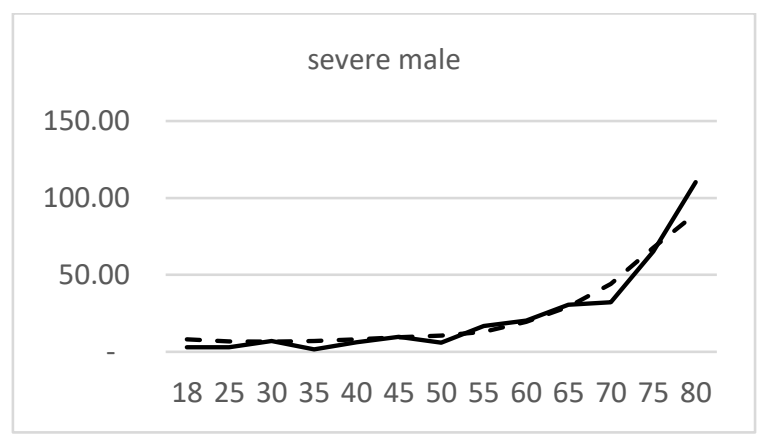

(d)

Figure 3. The comparison between the calculated WHS disability prevalence rates (solid lines) and the model's prevalence rate (dashed lines) for males with different disability levels: able (a), mild (b), moderate (c) and severe (d) 
Figure 2 and Figure 3 show further comparison between the data and the fitted model. The model prevalence rates (dashed lines) and the data prevalence rates (solid lines) are generally close to each other, particularly for able, mild and severe categories. For the mild level among female, we can see that the model's rates could not match the observed fluctuating trends that occur at later ages.

Note that the model's prevalence rates in Figure 2 and Figure 3 are obtained after we fitted the model and determined parameters. The estimated parameters involved in the formulas in equations 4 and 5 for $\operatorname{Newdis}(x)$ and Severity $(x, j)$ are shown in Table 6 and Table 7 . Using the estimated parameters and formulas, transition rate of disablement for the Malaysian population, particularly those in need long term care, can be calculated. These transition rates are useful and necessary to project the currently healthy and disabled population forward. Transitions include, for example, healthy people becoming disabled, disabled people becoming more severely disabled, and people dying.

Table 6. The estimated parameters for $\operatorname{Newdis}(x)$ formula for males and females

\begin{tabular}{|c|c|c|}
\hline Parameter & Males & Females \\
\hline$\alpha$ & 0.7888 & 0.8153 \\
\hline$A$ & 0.0097 & 0.0007 \\
\hline$B$ & 1.0598 & 1.0452 \\
\hline$C$ & 98.1510 & 109.3219 \\
\hline$D$ & 0.5382 & 0.6472 \\
\hline$E$ & 52.0239 & 42.9881 \\
\hline
\end{tabular}

Table 7. The estimated parameters for $\operatorname{Severity}(x, j)$ the formula for males and females

\begin{tabular}{|c|c|c|}
\hline Parameter & Males & Females \\
\hline$F$ & 0.4110 & 0.4221 \\
\hline$G$ & 1.0824 & 1.5060 \\
\hline$H$ & 81.5673 & 78.2006 \\
\hline$W(1)$ & 0.9654 & 0.9621 \\
\hline$W(2)$ & 0.3741 & 0.4287 \\
\hline$W(3)$ & 0.3342 & 0.2566 \\
\hline$I$ & 2.0998 & 3.7592 \\
\hline
\end{tabular}

We provide the estimated Malaysian disability transition rates for Malaysian elderly males and females as in Table 8 and Table 9. Although transition rates were calculated from age 18 years old for both males and females, we provide transition rates for the selected elderly group aged 60 years and above only.

We can see from the Table 8 and 9 that the chance or probability that a person to remain at current disability state next year (able to able, mild to mild, etc.) is the highest compared to probabilities that he deteriorates to worse disability states or improves to a better state. It is noteworthy that the probability that a severely disabled elderly to die next year is higher than those less severely disabled. For example, the probability that an 80 years old severely disabled male to die is $25.60 \%$ compared to $17.54 \%$ for those in other disability categories. Similarly, the probability that an 80 years old severely disabled female to die is $51.43 \%$ higher than those in different disability categories.

Table 8. Malaysian male disability transition rates for selected old age groups

\begin{tabular}{|c|c|c|c|c|c|}
\hline & Able & Mild & Moderate & Severe & Dead \\
\hline \multicolumn{6}{|c|}{ Able } \\
\hline 60 & 0.9339 & 0.0378 & 0.0073 & 0.0033 & 0.0177 \\
\hline 70 & 0.8787 & 0.0564 & 0.0127 & 0.0066 & 0.0457 \\
\hline 80 & 0.7092 & 0.0807 & 0.0215 & 0.0132 & 0.1754 \\
\hline \multicolumn{6}{|c|}{ Mild } \\
\hline 60 & 0.1441 & 0.8159 & 0.0154 & 0.0069 & 0.0177 \\
\hline 70 & 0.1374 & 0.7766 & 0.0266 & 0.0138 & 0.0457 \\
\hline 80 & 0.1147 & 0.6371 & 0.0451 & 0.0277 & 0.1754 \\
\hline \multicolumn{6}{|c|}{ Moderate } \\
\hline 60 & - & 0.1452 & 0.8226 & 0.0145 & 0.0177 \\
\hline 70 & - & 0.1390 & 0.7864 & 0.0289 & 0.0457 \\
\hline 80 & - & 0.1165 & 0.6499 & 0.0582 & 0.1754 \\
\hline \multicolumn{6}{|c|}{ Severe } \\
\hline 60 & - & - & 0.1150 & 0.80509 & 0.0799 \\
\hline 70 & - & - & 0.1099 & 0.76990 & 0.1201 \\
\hline 80 & - & - & 0.0930 & 0.65100 & 0.2560 \\
\hline
\end{tabular}

Therefore, based on the results, we believe that attention should be focused particularly to the severe disability category as it is the most important in the context of long-term care as acknowledged by Leung (2004). Severely disabled elderlies refer to those who are unable to perform the majority of activity daily livings hence needing round-the-clock care 
services. The costs for caring and treating the elderly with severe disability conditions are high and continue to increase. In America, the health care costs in the final phase of life, when living with chronic health conditions have the most intense costs and treatment (Lynn \& Adamson 2003). Thus, more studies are critically needed to prevent and alleviate disability severity, particularly to keep physically and mentally active when getting older or after retirement.

Table 9. Malaysian female disability transition rates for selected old age groups

\begin{tabular}{|c|c|c|c|c|c|}
\hline & Able & Mild & Moderate & Severe & Dead \\
\hline \multicolumn{6}{|c|}{ Able } \\
\hline 60 & 0.9346 & 0.0437 & 0.0082 & 0.0021 & 0.0114 \\
\hline 70 & 0.8865 & 0.0635 & 0.0125 & 0.0033 & 0.0342 \\
\hline 80 & 0.7296 & 0.0739 & 0.0268 & 0.0130 & 0.1567 \\
\hline \multicolumn{6}{|c|}{ Mild } \\
\hline 60 & 0.1425 & 0.8073 & 0.0309 & 0.0078 & 0.0114 \\
\hline 70 & 0.1363 & 0.7701 & 0.0469 & 0.0124 & 0.0342 \\
\hline 80 & 0.1076 & 0.5862 & 0.1006 & 0.0490 & 0.1567 \\
\hline \multicolumn{6}{|c|}{$\begin{array}{l}\text { Moder } \\
\text { ate }\end{array}$} \\
\hline 60 & - & 0.1439 & 0.81526 & 0.0294 & 0.0114 \\
\hline 70 & - & 0.1381 & 0.7810 & 0.0466 & 0.0342 \\
\hline 80 & - & 0.1032 & 0.5561 & 0.1840 & 0.1567 \\
\hline \multicolumn{6}{|c|}{ Severe } \\
\hline 60 & - & - & 0.1158 & 0.8106 & 0.0736 \\
\hline 70 & - & - & 0.1114 & 0.7799 & 0.1087 \\
\hline 80 & - & - & 0.0953 & 0.6674 & 0.2373 \\
\hline
\end{tabular}

\section{REFERENCES}

Bueno, LPV 2013, 'Brazilian long-term care plan: an evaluation based on the Singaporean model', MSc. Thesis, CASS Business School, City University London.

Chan, WS, Li, SH \& Fong, PW 2004, 'An actuarial analysis of long-term care demand in Hong Kong', Geriatrics and Gerontology International, vol. 4, pp. 143-145.

Continuous Mortality Investigation Bureau 1999, Continuous Mortality Investigation Reports, 17 edn. Continuous Mortality Investigation Bureau of the Institute of Actuaries. Department of Statistics Malaysia 2002, Abridged Life Tables, Malaysia, 2002-2004.

Goh, ZY \& Lai, MM 2013, 'The formal and informal long-term caregiving for the elderly: the Malaysian experience', Asian

\section{CONCLUSION}

This research provides the functional discrete Markov model methods to estimate transition rates of disablement for the Malaysian population, given that the longitudinal data is unavailable. The estimated parameters were found accurate as they can replicate the actual prevalence rates with sum squared errors, less than $5 \%$ for almost all age groups and disability states. The probability of elderlies to remain in his/her current state next year is the highest compare to deteriorate or improve to other states. Severely disabled elderlies, especially the oldest age group, have the highest risk to die compared to less severely disabled elderlies. The model and outputs of this paper are significant for future research. More works are needed particularly to project the number of people who likely need long term care and its related costs.

\section{ACKNOWLEDGEMENTS}

The author is thankful for research funds received from the Ministry of Education (MOE) and Institute of Research Management Centre (RMC), Universiti Teknologi MARA (UiTM) through the Fundamental Research Grant Scheme with the file number of 6oo-IRMI/FRGS 5/3 (125/2019).

Social Science, vol. 9, NO. 4, pp. 174-184.

Hariyanto, EA, Dickson, DCM \& Pitt, DGW 2013, 'Estimation of disability transition probabilities in Australia I: Preliminary', Annals of Actuarial Science, vol. 8, no. 1, pp. 131-155.

Hyndman, RJ 2010, ADDB: Australian demographic data bank, R package version 3.223'.

URL: http://robjhyndman.com/software/addb/

Lee, RD \& Carter, LR 1992, 'Modelling and forecasting U.S mortality', Journal of the American Statistical Association vol. 87 , no. 419 , pp. 659-671.

Leung, E 2004, 'Projecting the needs and costs of long-term care in Australia', Australia Actuarial Journal, vol. 10, no. 
2, pp. 343-385.

Lynn, J \& Adamson, DM 2003, Living well at the end of life,

RAND Health.

Manton, KG 2010, 'National Long-Term Care Survey: 1982, 1984, 1989, 1994, 1999, and 2004', in Ann Arbor, MI: Interuniversity Consortium for Political and Social Research. https://doi.org/10.3886/ICPSRo9681.v5.

Omar, MH, Shair, SN \& Asmuni, NH 2017, 'Lung cancer transition rate by stages using discrete time Markov model', Indonesian Journal of Electrical Engineering and Computer Science, vol. 18, no. 3, pp. 1,295-1,302.

Pollard, J 1996, 'On the changing shape of the Australian mortality curve', Health Transition Review, vol. 6(Supplement), pp. 283-300.

Rickayzen, BD \& Walsh, DEP 2002, 'A multi-state model of disability for the United Kingdom: implications for future need for long term care for the elderly', British Actuarial Journal, vol. 8, pp. 341-393.

Society of Actuaries Long-Term Care Valuation Insurance Methods Task Force 1995, 'Long-term care valuation insurance methods', Transactions of Society of Actuaries, vol. 47, pp. 103-271.

Tareque, MI, Tiedt, AD, Islam, TM, Begum, S \& Saito, Y 2017, 'Gender differences in functional disability and self-care among seniors in Bangladesh', BMC Geriatrics, vol. 17, no. 1 , p. 177.

WHS 2002, The World Health Survey, World Health Organization (WHO).

URL: http://www.who.int/healthinfo/survey/ 